\title{
DOES COLPOSCOPICALLY DIRECTED PUNCH BIOPSY REDUCE THE INCIDENCE OF NEGATIVE LLETZ?
}

\author{
LYNETTE ANN DENNY MBChB (UCT) FCOG (SA) \\ Fellow in Gynaecological Oncology \\ Groote Schuur Hospital \& University of Cape Town
}

A dissertation submitted to the University of Cape Town in fulfilment of the requirements for the degree of Master of Medicine (Obstetrics \& Gynaecology) Part III 
The copyright of this thesis vests in the author. No quotation from it or information derived from it is to be published without full acknowledgement of the source. The thesis is to be used for private study or noncommercial research purposes only.

Published by the University of Cape Town (UCT) in terms of the non-exclusive license granted to UCT by the author. 


\section{DECLARATION}

LYNETTE DENNY hereby declare that the work on which this thesis is based is my original work (except where acknowledgements indicate otherwise) and that neither the whole work nor any part of it has been, is being, or is to be submitted for another degree in this or any other University.

I empower the University to reproduce for the purpose of research either the whole or any portion of the contents in any manner whatsoever.
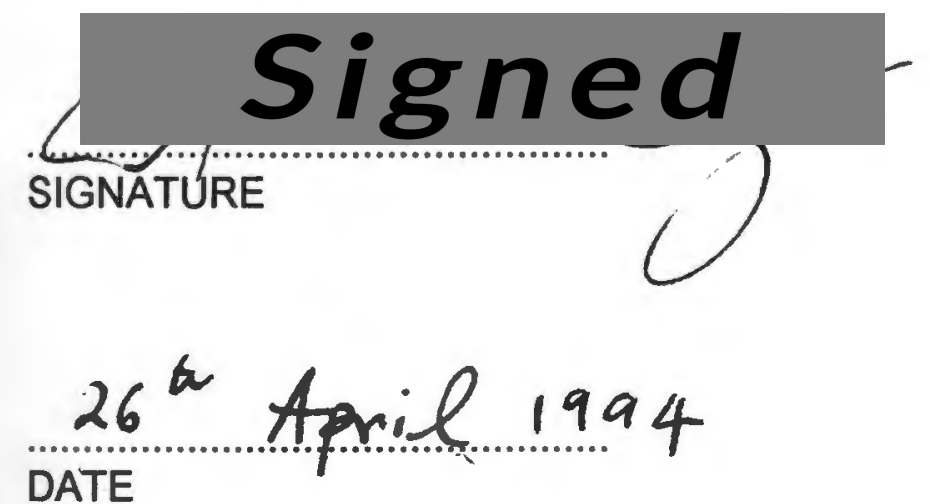


\section{ACKNOWLEGEMENTS}

Mrs Nazli Dollie and Sister Janine Jones of the Colposcopy Clinic provided invaluable assistance in the search for and retrieval of patient files. Their committment to the clinic and research is deeply appreciated.

Mr Freddie Lotters assisted in retrieving literature from the library, for which I am most grateful.

Thank you to the Oncology Unit, in particular Dr Katrien Dehaeck, Professor Basil Bloch and $\mathrm{Dr}$ James Nevin for reading the dissertation and offering constructive ideas and suggestions.

Ms Nicole Ihle typed and organised the manuscript with her characteristic flair, attention to detail and perfectionism.

Finally sincere gratitude to my supervisor, Dr Robbert Soeters whose patience, support and encouragement made this dissertation possible. 


\section{TABLE OF CONTENTS}

Pg. No.

$\begin{array}{llr}\text { CHAPTER } 1 & \text { INTRODUCTION } & 1 \\ \text { CHAPTER 2 } & \text { AIMS OF THIS STUDY } & 5 \\ \text { CHAPTER 3 } & \text { MATERIALS \& METHODS } & 7 \\ \text { CHAPTER 4 } & \text { RESULTS } & 15 \\ \text { CHAPTER 5 } & \text { DISCUSSION } & 23 \\ \text { CHAPTER 6 } & \text { CONCLUSION } & 31 \\ & \text { REFERENCES } & 32\end{array}$




\section{LIST OF TABLES}

I Referring cytology of study group

II Referring cytology of negative LLETZ in group B

III Referring cytology in Group A with negative follow-up cervical smears

IV Calculation of sensitivity and specificity

V Summary of calculations 


\section{LIST OF FIGURES}

1 Protocol for management of an abnormal cervical smear

2 Protocol for "look \& LLETZ"

3 Results for LLETZ and prior punch biopsy

$4 \quad$ Results for "look \& LLETZ" group 18

$5 \quad$ Negative punch biopsy group 


\section{CHAPTER 1}

\section{INTRODUCTION}

Cervical Intraepithelial Neoplasia (CIN) is recognised as a precursor to invasive cancer of the cervix. 1,2,3,4 Well organised screening programmes have been shown to substantially reduce the incidence of and mortality from cervical cancer in the screened population provided adequate facilities exist for treatment and follow-up of affected individuals. 5,6 In Iceland a more than two-fold reduction in mortality from cervical carcinoma was observed in the screened population of women aged 25 - 59 years, between 1965 and 1974.6

Prior to the introduction of colposcopy, the standard diagnostic procedure for an abnormal papanicolaou smear of the cervix was cone biopsy. Cone biopsy removes the entire Transformation Zone (TZ) of the cervix (from where over $95 \%$ of cervical cancers arise) and provides a histological sample for evaluation. This procedure has been associated with well recognised short and long term morbidity, including primary and secondary haemorrhage, infertility due to cervical stenosis, cervical incompetence and preterm labour. $7,8,9,10$ In addition the procedure requires admission to hospital and is performed under general anaesthesia.

In an attempt to reduce the use of cone biopsy, the diagnostic procedure of random multiple quadrant biopsies of the cervix was introduced. Numerous published reports have confirmed the diagnostic inaccuracy and high false negative rate of this method of evaluating the cervix. $11,12,13$

Colposcopy, introduced by Hinselman in 1926, brought a new dimension to the management of an abnormal cytological smear of the cervix. Visual inspection of the illuminated and magnified cervix through the colposcope enabled: 
1. The presence of the lesion to be confirmed.

2. The size, extent and severity of the lesion to be determined.

3. The exclusion of invasive disease.

While colposcopy provided a clinical evaluation of the cervix, cytology examined the morphologic changes in the exfoliated cell. Initially these two modes of assessment of the cervix were seen as competitors, however, studies comparing cytology with colposcopy showed that the combination of both methods significantly increased diagnostic accuracy over that of each method used separately. $14,15,16$

The use of cytology and/or colposcopy alone has traditionally been considered an inadequate basis for surgical therapy of $\mathrm{CIN}$. Neither cytology nor colposcopy were considered to be final diagnostic techniques. They were seen to be tools for the pretreatment assessment of the cervix that allowed the identification, extent and severity of the lesion to be defined. They did not provide a histological diagnosis.

To improve diagnostic accuracy, colposcopically directed punch biopsy from the most abnormal area, as òpposed to the earlier practice of random biopsies, was developed.14,15,16 With the information provided by a combination of cytology, a satisfactory colposcopy and colposcopically directed punch biopsy, conservative methods for the treatment of CIN were introduced. These methods consisted of destructive or ablative therapy of the $T Z$, as opposed to the more radical surgical treatments that had previously been used.

The most obvious advantage of local ablative methods over the traditional cone biopsy were their simplicity - most could be performed under local anaesthesia as outpatient procedures, with minimal short or long term side-effects. 
Over time, five different ablative techniques were introduced for the local, outpatient management of $\mathrm{CIN}$. These were cryosurgery, electrocoagulation diathermy, electrocautery (which required general anaesthesia), cold coagulation and $\mathrm{CO}_{2}$ laser vaporisation. The greatest degree of success has been reported by those using laser, with cure rates for CIN in excess of $94 \% .17,18,19,20$

Ablative methods for the treatment of CIN have dominated practice for the past 15 years. Central to the use of ablative methods has been expert colposcopy and histological confirmation of the cytological and colposcopic diagnosis with punch biopsy. The use of ablative methods have however not been without considerable controversy, the most important being that histological confirmation of the lesion relies on the diagnosis made by punch biopsy.

Colposcopically directed punch biopsy has been shown to be unreliable in a number of studies.21,22,23,24 In addition, the confidence with which invasive disease can be excluded depends on the colposcopist correctly identifying the most abnormal area for biopsy. In other words, the punch biopsy is only as good as the colposcopist.

A major concern connected with physical methods of therapy has been the risk of inadequate destruction of lesions deep within cervical crypts or glands. If the destruction is too shallow there is a risk that regenerating epithelium may cover residual neoplastic tissue allowing the development of occult invasive cancer. ${ }^{25}$ The work of Anderson et al ${ }^{26}$ showed that destruction of tissue to a depth of $3.38 \mathrm{~mm}$ would eradicate $99.7 \%$ of $\mathrm{CIN}$ 3 lesions. Abdul Karim et $a^{27}$ showed that a depth of destruction to $4.8 \mathrm{~mm}$ would eradicated the same percentage of CIN3 lesions.

Of greater concern is the possibility of missing microinvasive disease and/or preinvasive glandular lesions of the endocervix. The risk of developing invasive carcinoma after treatment for $\mathrm{CIN}$ has been reported as 2.2 to 2.4 per 1000 after laser ${ }^{25}, 3$ per 1000 after 
cone biopsy and 4 per 1000 after hysterectomy 28 . None of these comparative Figures are statistically different.

In a review of 26 patients with microinvasive tumours, Choo et al 29 found that only $26 \%$ were correctly visualised as microinvasive at colposcopy. In addition four of the 22 patients in which the TZ was seen in its entirety and who had punch biopsies, the punch biopsy failed to detect microinvasion, subsequently diagnosed by cone biopsy or hysterectomy.

McIndoe et al ${ }^{30}$ noted a fall in the rate of diagnosis of microinvasive disease following the introduction of ablative treatment, suggesting that unrecognised tumors were being destroyed. They advised a return to treatment of CIN by excision. In their study of 119 women who had punch biopsies taken prior to laser conisation of the cervix, there were two undiagnosed microinvasive lesions. Sixteen women had lesions two or three grades worse than predicted by punch biopsy.

In 1981 Cartier $^{31}$ described taking biopsies and excising the TZ of the cervix in strips using a low voltage diathermy loop. In 1986, Prendiville et al32 compared the low voltage diathermy loop for taking cervical biopsies with punch biopsy forceps and showed that the quality of biopsies obtained for histological analysis using the loop diathermy were far superior to those taken with punch biopsy forceps.

In 1989 this technique was further developed by Prendiville to excise the entire TZ, rather than performing a limited punch biopsy, and was called Large Loop Excision of the Transformation Zone or LLETZ ${ }^{33}$. In America the acronym LEEP (Loop Electrosurgical Excisional Procedure) was adopted. 


\section{CHAPTER 2}

\section{AIMS OF THIS STUDY}

LLETZ has gained widespread acceptance on the basis that it is an excisional technique, performed as an outpatient procedure, under local anaesthetic. Required equipment is inexpensive and the procedure easy to learn and simple to perform. Morbidity is low although long term follow-up studies are awaited. In addition cure rates comparable to those achieved with ablative treatment have been documented. $34,35,36,37,38$

Many centres have taken LLETZ a step further and introduced the so-called look and LLETZ. $34,36,37$ The patient is treated for CIN diagnosed on cytological and colposcopic assessment of the cervix alone, without the use of pre-treatment punch biopsy.

A disturbing aspect of this approach is the frequency with which no CIN is diagnosed in the final LLETZ specimen - the so-called negative LLETZ, which ranges from 5 to $35 \% .34,36,37,38$ A negative LLETZ implies that both the original cytology and colposcopy were falsely positive and the women were subjected to an apparently unnecessary surgical procedure. Alternatively it may indicate that the lesion was missed by the LLETZ procedure.

LLETZ was adopted as the preferred mode of treatment of CIN in the Colposcopy Clinic at GSH in July 1991. In view of the concerns about negative LLETZ we examined our data base in order to ascertain:

1. Our own incidence of negative LLETZ. 
2. Whether the use of punch biopsy prior to treatment by LLETZ resulted in a lower incidence of negative LLETZ versus that occuring in the look and LLETZ group in our unit.

3. Whether patients undergoing punch biopsy would be saved from a LLETZ procedure because of the information provided by the punch biopsy. 


\section{CHAPTER 3}

\section{MATERIALS AND METHODS}

The study group was drawn from a cohort of new patients referred to the Colposcopy Clinic at Groote Schuur Hospital between July 1991 and December 1992. Patients attending with previously treated or recurrent disease were excluded from the study.

Referral criteria to the colposcopy clinic are:

1. a single pap smear showing $\mathrm{CIN} 2$ or 3 ,

2. two smears repeated at six month intervals showing $\mathrm{CIN} 1$

Patients are referred to the clinic by the Cytology Laboratory, Department of Pathology, University of Cape Town. This laboratory examines all pap smears performed on patients seen in the geographical area served by Groote Schuur Hospital. Patients with abnormal smears are traced through a centralised service. The women are sent letters or traced through a network of district Sisters after which an appointment is made for the patient to attend the Colposcopy Clinic. If the patient fails to arrive for an appointment, attempts to trace her are continued for up to one year.

Five hundred and ninety six women with abnormal papanicolaou smears of the cervix fulfilling the above criteria were referred to the Colposcopy Clinic during the study period. All patients who were treated with LLETZ were entered onto a computerised data base using DBASE 3. Information on the remaining patients was retrieved from the clinic files.

Seventy eight women (13\%) underwent cone biopsy as they were deemed unsuitable for local therapy. Indications for cone biopsy included: 
1. Inability to visualise the entire lesion colposcopically.

2. The suspicion of microinvasion on either cytology or colposcopy.

3. The absence of a colposcopic lesion in the presence of persistently abnormal cytology.

Invasive carcinoma of the cervix was diagnosed in 36 women (6\%). Depending on stage these patients were referred either for surgery or radical radiotherapy.

One hundred and twenty seven women $(21 \%)$ either failed to arrive for their first appointment or came only once and were then lost to follow-up. Twenty nine women (5\%) were found to have a normal cervix on colposcopic examination and subsequently had two to three negative follow up smears. These patients were presumed to represent false positive cytology and were discharged from the clinic.

Three hundred and twenty six women referred to the clinic with CIN on pap smear were considered suitable for local treatment of the lesion (55\%). Of this group 28 women (9\%) were treated with laser ablation as the necessary equipment for LLETZ was not available on the day they arrived for treatment. These women are excluded from further analysis, leaving a study group of 298 women.

Colposcopic examinations were performed by a group of 4 consultants with experience ranging from 5 to 10 years, as well as by senior registrars with one year's supervised experience. Patients were examined and, if considered suitable for LLETZ were treated in the colposcopy clinic.

The indications for LLETZ were the same as those for laser ablation of the cervix. At Groote Schuur Hospital, prior to July 1991, the preferred outpatient management of patients with CIN was laser ablation of the TZ. All patients with CIN on pap smear were examined colposcopically. The lesion was considered suitable for local therapy if the following criteria were satisfied: 
1. A lesion was confirmed colposcopically.

2. The entire extent of the lesion was visible both on the ecto- and in the endocervix and the lesion did not extend beyond the ectocervix.

3. There was no evidence of microinvasive or invasive disease.

If these criteria were fulfilled, a punch biopsy was taken from the area of the cervix judged most abnormal with good correlation between cytology, colposcopy and histology laser ablation of the TZ to a depth of at least $5 \mathrm{~mm}$ was performed, as described by Jordan et al'.

In July 1991 LLETZ was introduced for the treatment of CIN suitable for local therapy. The patient was examined colposcopically, a punch biopsy was taken and the patient was managed according to the following protocol:

1. If the lesion was confirmed histologically and there was no evidence of invasion, the patient was referred for LLETZ at a separate visit.

2. If the punch biopsy was negative, the patient was recalled for a repeat cytological smear. If the repeat smear at three months was positive for $\mathrm{CIN}$, then she was referred for LLETZ regardless of the punch biopsy result.

3. If the repeat cytological smear at three months was negative, a third smear six months later was performed. If this remained negative for CIN at nine months, routine cytological follow-up was instituted.

4. If the smear taken at nine months was positive, a repeat colposcopy was performed (Figure 1).

As experience with this new technology was gained, two of the four most experienced consultants stopped performing punch biopsies. These patients were referred for LLETZ solely on the basis of abnormal cytology and colposcopy and without histological confirmation by punch biopsy (Figure 2). The other members of the clinic continued to 


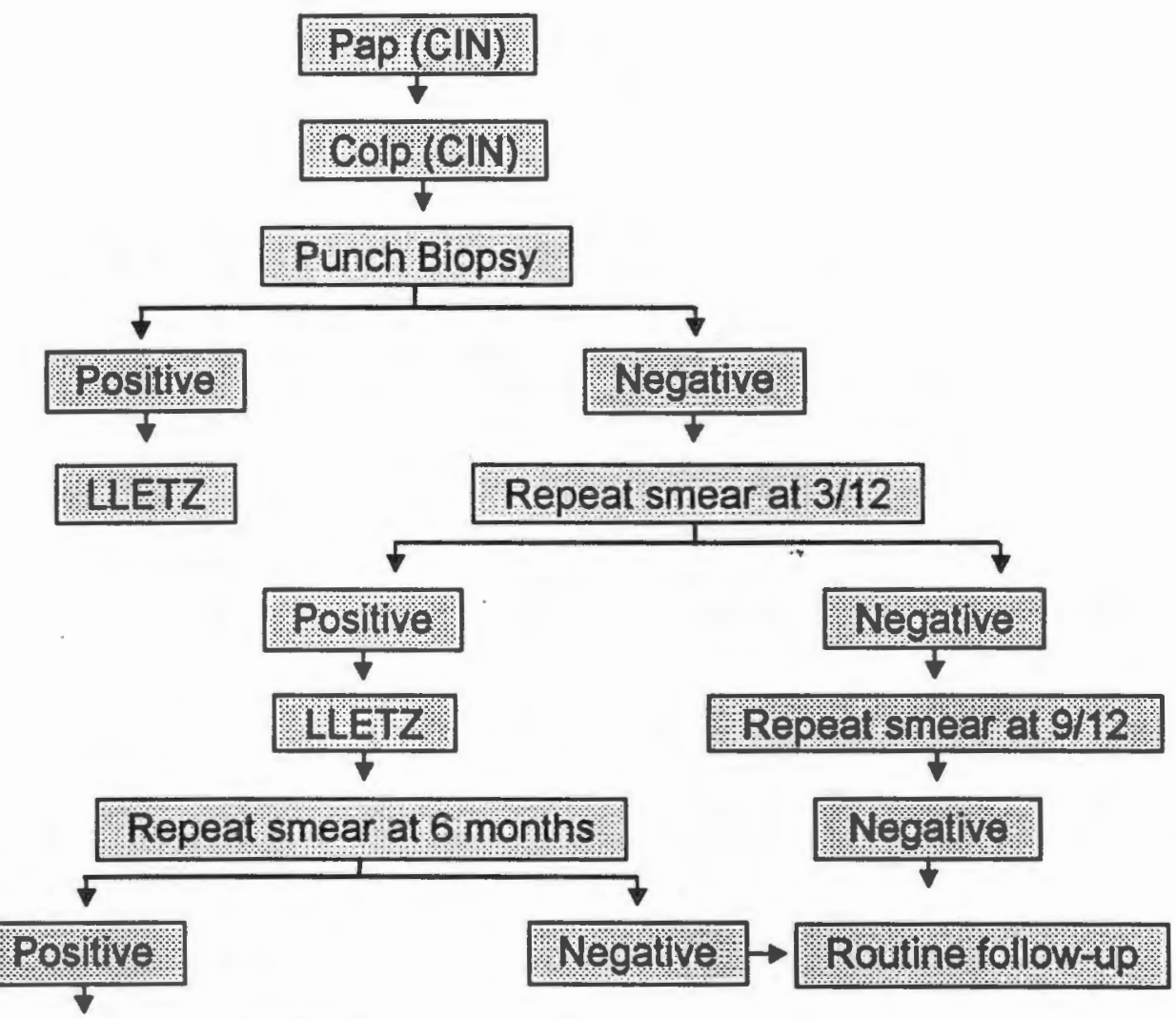

Reevaluation appropriate $\mathrm{RX}$

Figure 1. Protocol for management of an abnormal cervical smear 


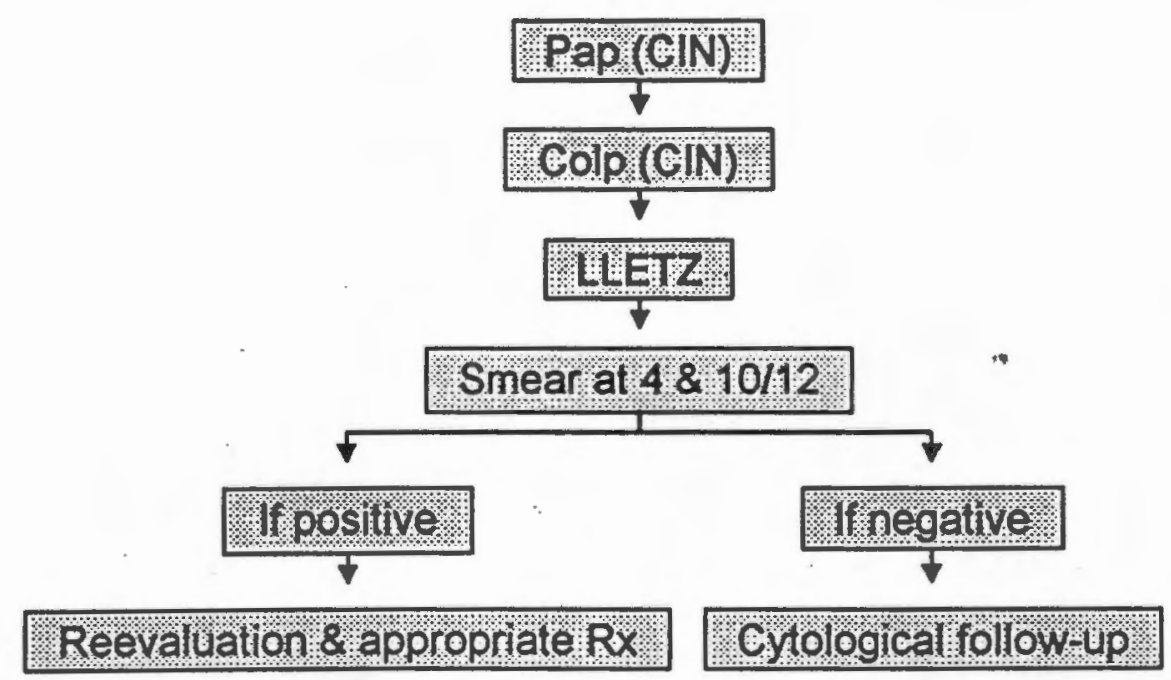

Figure 2. Protocol for "look and LLETZ" 
obtain histological confirmation by punch biopsy. Thus the decision as to whether to perform a punch biopsy or not was an individual one, based on colposcopic experience and was neither randomised nor part of a pre-planned protocol.

We were thus able to identify two groups of patients, Group A and Group B. Group A represented a group of 184 women referred with CIN on pap smear, confirmed colposcopically and who underwent punch biopsy.

Group B consisted of 114 women referred for LLETZ on the basis of abnormal cytology, confirmed on colposcopy alone - so-called look and LLETZ.

Cervical intraepithelial neoplasia was graded as CIN 1 for mild dysplasia, CIN 2 for moderate dysplasia and CIN 3 for severe dysplasia.

Large Loop Excision of the Transformation Zone involves using a thin stainless steel wire loop of various dimensions which is connected to an insulated holder. This is connected to an electrosurgical unit which uses a blend of cutting and cautery at low output levels. Most hospitals are alrealdy equipped with standard electrosurgical units used routinely for diathermy haemostasis or cutting at surgery. It was thus not necessary to invest in any new equipment other than the wire loops.

The procedure was performed in the colposcopy clinic where the cervix was stained with Lugols lodine using a $5 \mathrm{ml}$ syringe with which the cervix was sprayed. A commercial preparation containing $3 \%$ prilocaine hydrochloride and $0.03 \%$ octapressin (Citonest) was used as local anaesthetic. This was infiltrated into the cervical stroma beneath and around the TZ using a dental syringe and needle.

The procedure was performed under colposcopic guidance. Starting about $0.5 \mathrm{~mm}$ outside the $\mathrm{TZ}$, the loop was advanced inwards and vertically until the required depth of at least $5 \mathrm{~mm}$ was reached. The loop was then slowly taken from right to left (or vice 


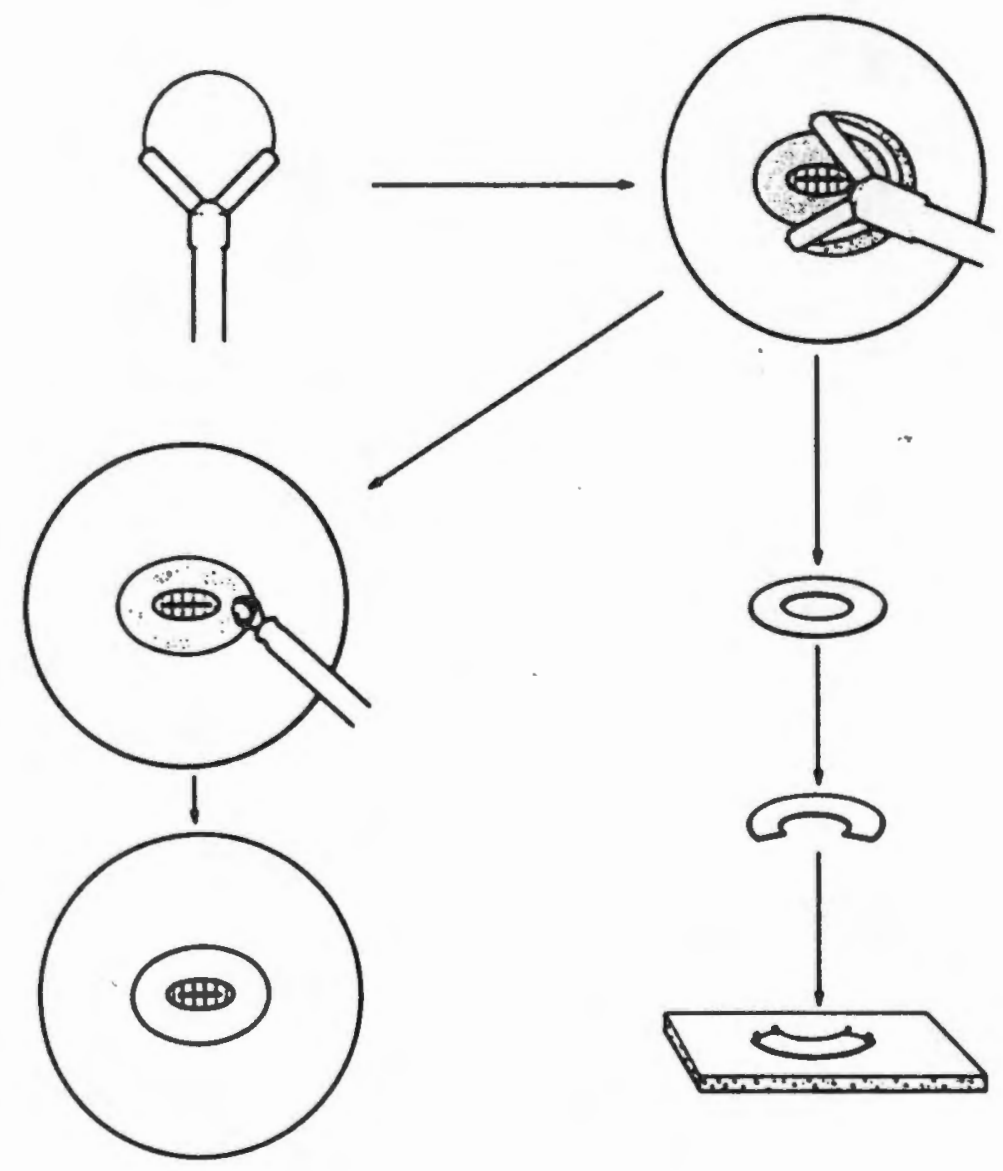

Diagrammatic illustration of the LLETZ procedure 
versa) across the cervix beneath the $T Z$. It was then withdrawn $0.5 \mathrm{~mm}$ beyond the opposite lateral margin of the TZ, allowing excision of the TZ in one piece. Some colposcopists preferrred to remove the TZ from top to bottom.

Haemostasis of the remaining crater in the cervix was obtained using ball diathermy to the base in conjunction with ferric subsulphate (Monsels') solution or silver nitrate. Thereafter a tampon coated with sulphasalasine (Nelex) cream was inserted into the crater and the patient advised to remove it six hours later.

Different loop sizes and shapes enabled the colposcopist to choose the most apppropriate loop for the individual variation in size and shape of the TZ. Where the TZ could not be removed in one scoop, either a wider or deeper wire.loop was chosen or the TZ was removed in two or more pieces. After excision the tissue was pinned onto a piece of cork and reconstructed to facilitate correct orientation of the specimen for histological assessment. The specimen was then placed in formalin for preservation and transport to the laboratory. 


\section{CHAPTER 4}

\section{RESULTS}

The study group consisted of 298 new patients referred to the colposcopy clinic with cytological and colposcopic evidence of CIN.

The mean age of the study group was 32.4 years, with a range of 19 to 58 years. Reasons for referral are shown in Table I:

Table I. Referring cytology of study group

\begin{tabular}{|c|c|c|}
\hline Persistent CIN 1 & 41 & $(13.76 \%)$ \\
\hline CIN 2 & 79 & $(26.5 \%)$ \\
\hline CiN 3 & 178 & $(59,73 \%)$ \\
\hline
\end{tabular}

Group A consisted of 184 women (61.74\% of the study group) who had CIN diagnosed cytologically and colposcopically and who underwent a colposcopically directed punch biopsy. Of one hundred and twenty three women who had CIN demonstrated in the punch biopsy 116 underwent the LLETZ procedure, and seven (5\%) failed to arrive for their appointment and were lost to follow up.

Of the 116 women, 95 had CIN confirmed in the LLETZ histology (81.89\%) and 21 patients had no CIN identified (18.10\%) (see Figure 3).

Group B, as described above, the look and LLETZ group, consisted of 114 women (38\% of the study group). Ninety seven of these women had CIN confirmed on histology of the 


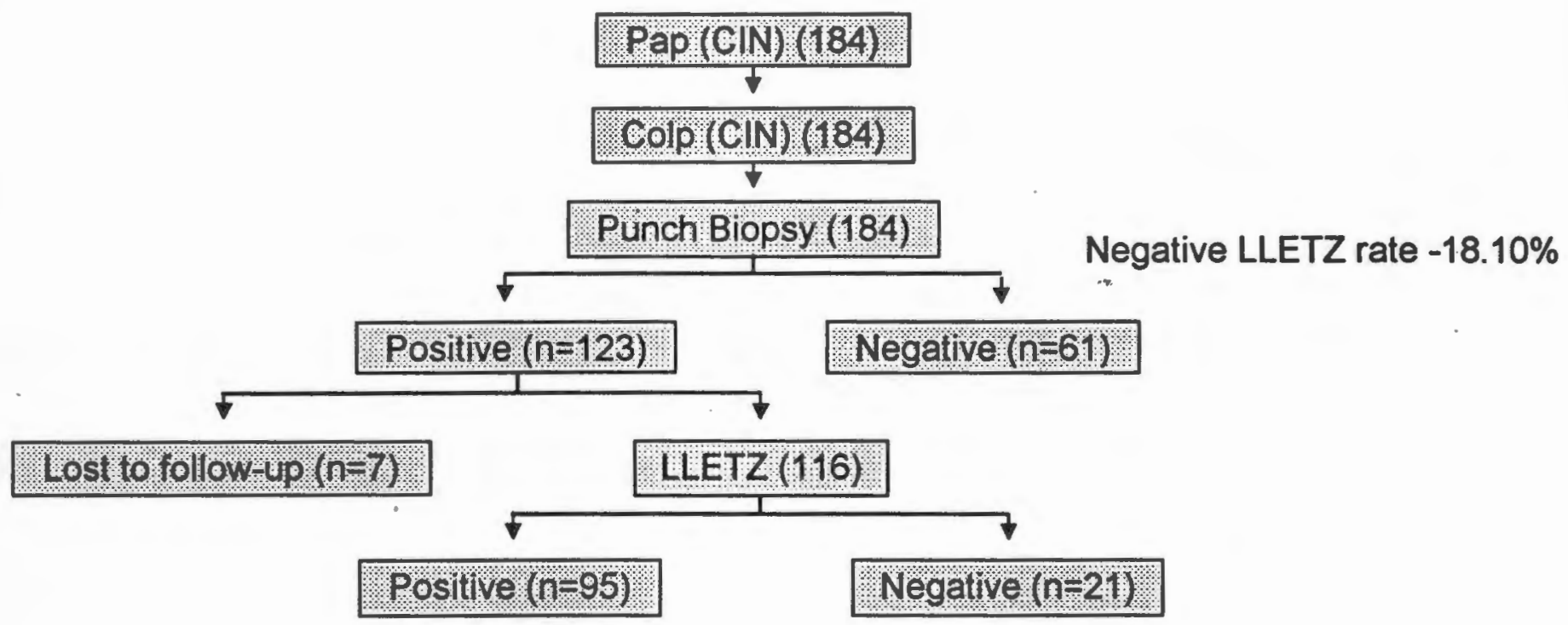

Figure 3. Results for LLETZ and prior punch biopsy 


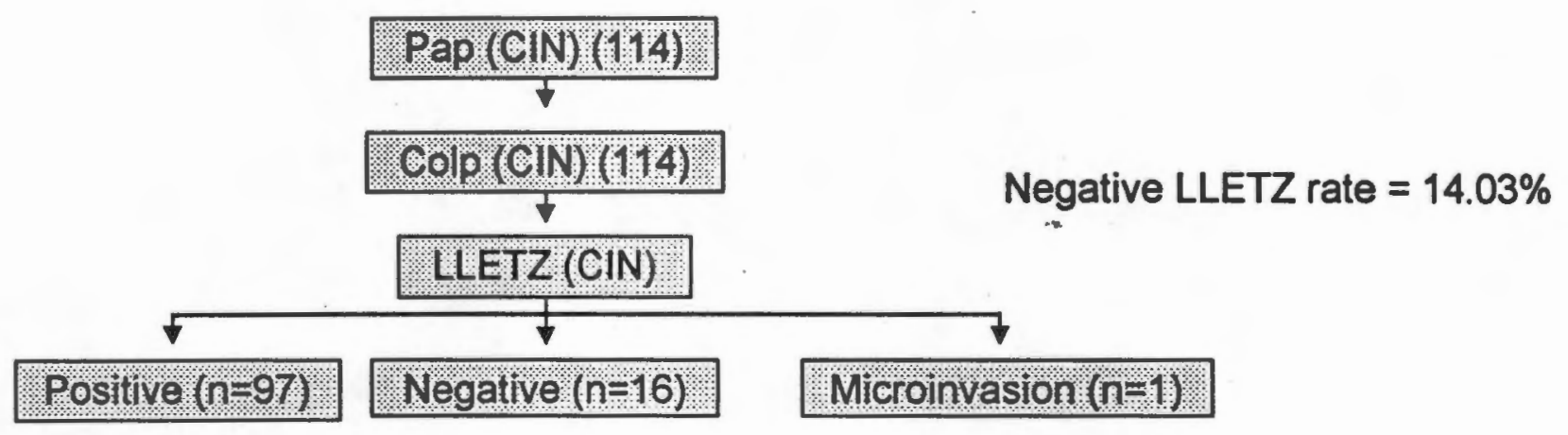

Figure 4. Results for "look and LLETZ" group 


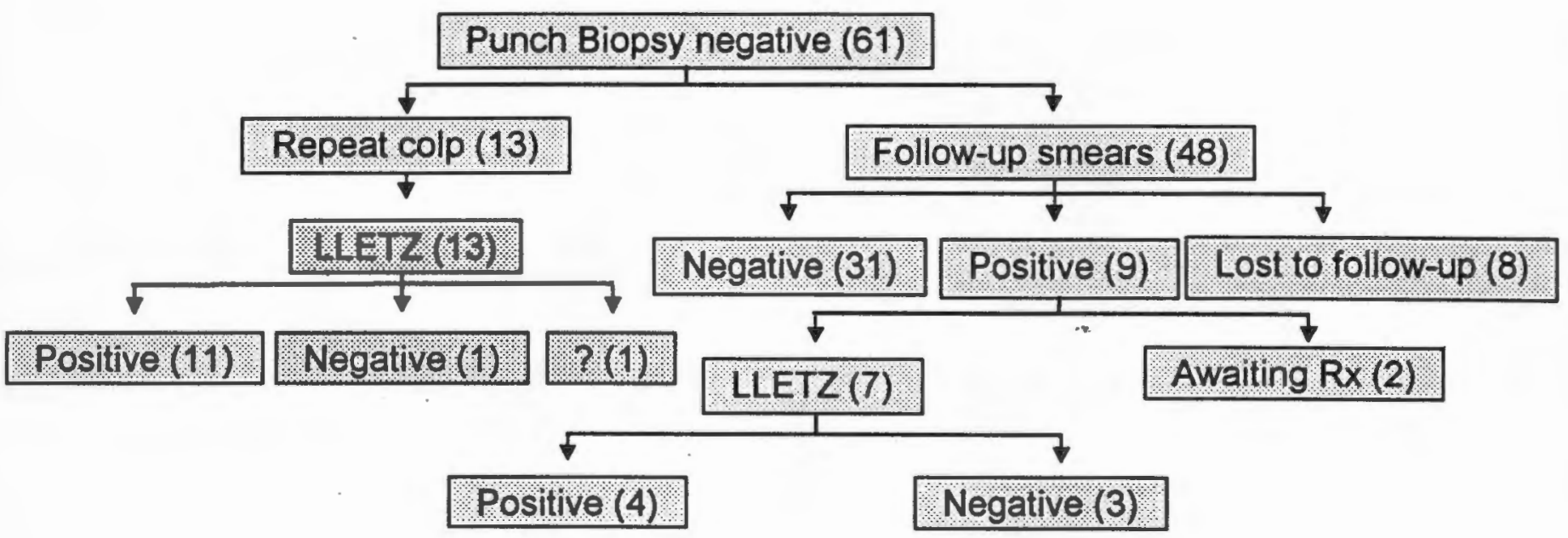

Figure 5. Negative Punch Biopsy Group 
Forty eight women with negative punch biopsies were followed up cytologically $(79 \%)$. Nine women had positive follow up smears $(19 \%=9 / 48)$. Of these, 7 had LLETZ, of which 3 were negative for CIN and 4 had CIN confirmed in the final LLETZ histology. Two women defaulted but have subsequently been traced and at the time of writing are awaiting treatment. Eight women in this group of $48(16.6 \%)$ failed to return for repeat smears and have subsequently been lost to follow up (Figure 5).

Thirty one of the women with negative punch biopsies were followed up cytologically and had two or more negative follow up smears, repeated at three months and then six months later (Figure 5). This group, $16.8 \%$ of group A, had referring cytology as indicated in Table III.

Table III. Referring cytology in Group A with negative follow-up cervical smears.

\begin{tabular}{|c|c|c|}
\hline $\mathrm{CIN} 3$ & $19 / 31$ & $(61.29 \%)$ \\
\hline $\mathrm{CIN}_{2}$ & $7 / 31$ & $(22.58 \%)$ \\
\hline CIN 1 . & $5 / 31$ & $(16,12 \%)$ \\
\hline
\end{tabular}

To calculate the sensitivity and specificity of punch biopsy Table IV was constructed making use of a number of assumptions. The gold standard against which punch biopsy has been calculated is the final LLETZ histology and those women with two or more negative plus fully representative follow-up smears. The assumption was made that two or more negative follow up smears would result in negative histology if the patient were subjected to LLETZ. 
Table IV Calculation of sensitivity and specificity

\begin{tabular}{|c|c|c|c|}
\hline CIN & LLETZ+ & LLETZ. & TOTAL \\
\hline $\mathrm{PB}+$ & 95 & 21 & 116 \\
\hline PB- & 15 & 35 & 50 \\
\hline TOTAL & 110 & 56 & 166 \\
\hline
\end{tabular}

The 15 patients who had negative punch biopsies followed by LLETZ include the 11 women who had a positive LLETZ after an initially negative punch biopsy but a second colposcopy that convincingly demonstrated $\mathrm{CIN}$. The other three women had a positive LLETZ after a negative punch biopsy followed by CIN that was demonstrated in follow up pap smears.

The 35 patients in the negative punch biopsy and negative LLETZ column includes the 31 women who had persistently negative smears after a negative punch biopsy. Three women had LLETZ after a negative punch biopsy but a positive follow up smear. One woman had a negative LLETZ after a negative punch biopsy but a repeat colposcopy that was convincingly positive.

Using the assumptions as detailed above the sensitivity and specificity of punch biopsy was calculated as follows:

- Sensitivity (true positives/true positives + true negatives) $=86.36 \%$

- Specificity (true negatives/true negatives + false positives) $=61.81 \%$.

- Positive Predictive Value of a positive punch biopsy (true positives/ total no. of positive tests) $=81.89 \%$

- Negative Predictive Value of a negative punch biopsy (true negatives/total no. of neg tests) $=68 \%$. 
These findings are summarised in table V:

TABLE V. Summary of Calculations

\begin{tabular}{|c|c|}
\hline & PUNCH BIOPSY \\
\hline SENSITIVITY & 86.36 \\
\hline SPECIFICITY & 61.81 \\
\hline PV (POS) & 81.89 \\
\hline PV (NEG) & 68 \\
\hline
\end{tabular}

PV $=$ Predictive value

The punch biopsy was falsely negative in 15 cases, representing $24.59 \%$ of all negative punch biopsies. 


\section{CHAPTER 5}

\section{DISCUSSION}

All modalities used in the diagnosis of CIN rely on subjective criteria, including cytology, colposcopy and histology. No one modality of assessment will ever be $100 \%$ accurate. Cervical intra-epithelial neoplasia is a disease largely of women of reproductive age. Treatment of this condition whether by ablation or excision causes mutilation of the cervix with potential loss of reproductive capacity.

It has been well established that CIN represents pre-invasive cancer of the cervix and treatment of this condition will prevent the development of cancer. The best modality of treatment has been the subject of a great deal of research and the pendulum has moved from excisional techniques, to ablative and now once again to excision of the disease.

This study has concerned itself with an audit of a new technology and a new approach to the treatment of pre-invasive neoplasia of the cervix. Our focus has been on those patients treated with LLETZ either with or without a preceding colposcopically directed punch biopsy. Treating patients with a surgical technique based on cytological and colposcopic criteria reflects a substantial departure from traditional surgical principles which emphasise that no patient should undergo a surgical procedure without prior histological confirmation of the lesion to be removed.

The introduction of a new technology requires vigorous audit to ensure that it is both effective and to the benefit of the patient. In addition, it is essential to adapt the new technology to the circumstances under which one is practicing. The look and LLETZ approach has considerable appeal in South Africa where both the attendance and follow up of patients with pre-invasive disease is unpredictable and subject to a high default 
rate. In our clinic $21 \%$ of all women referred with abnormal cervical smears to the Colposcopy Clinic at Groote Schuur Hospital defaulted.

A further advantage of combining diagnosis and treatment of $\mathrm{CIN}$ in one visit is the reduction in clinic visits and cost of travel to the hospital for the patients.

In this study no patients treated with look and LLETZ were lost to follow-up versus $8.5 \%$ of those women who had a punch biopsy and were booked for a second appointment.

These obvious advantages of the look and LLETZ procedure should not override the fact that it is contrary to acceptable medical practice to perform a medical procedure without the correct indications. It is assumed that a negative LLETZ is unnecessary and this should be avoided for two other reasons:

1. Most studies have reported morbidity of less than $5 \% 34,35,36$ but long term studies are not yet available and the impact of LLETZ on future fertility remains to be determined. There has been one reported case of cervical stenosis causing infertility after LLETZ42.

2. The very simplicity of the procedure lends itself to over-use or worse, abuse. The inappropriate use of LLETZ will only serve to further increase the occurence of negative LLETZ

This study showed that $14.03 \%$ of women treated with Look and LLETZ had no CIN diagnosed in the final LLETZ specimen despite positive cytology and colposcopy.

In the study by Murdoch et al38 $32.8 \%$ of their patients treated with look and LLETZ had no $\mathrm{CIN}$ on histological examination. Fifty three per cent of these women had decision cytologies of mild dysplasia (CIN 1) and their recommendation was to perform confirmatory punch biopsy in this group. On the other hand Bigrigg et al ${ }^{36}$ reported on 
negative histology in 47 out of $896(5 \%)$ look and LLETZ procedures. The decision cytology in the negative LLETZ group was either CIN 1 or CIN 2.

Our data showed that the decision cytology in the negative LLETZ group was CIN 1 in $44 \%$ of cases and we would concur with Murdoch et al ${ }^{38}$ that performing confirmatory punch biopsy in this group is indicated. Differentiating wart virus infection from low grade CIN colposcopically requires considerable expertise and may cause diagnostic confusion 39 .

However, it is disturbing that $56 \%$ of patients with negative LLETZ had CIN 2 or 3 on cytology and colposcopy, suggesting that both cytology and colposcopy were falsely positive.

It is possible that the lesion was situated deep in the crypts of the cervix and the lesion was missed by the excision. This however would not explain the fact that colposcopic examination had identified the lesion and was considered adequate at the time of examination.

Colposcopy is a skill that may require many years of training. According to Staff 40 there are three levels of expertise:

1. the ability to recognise the lesion, which in his opinion takes 3 to 4 months of training

2. the ability to sample by directed biopsy the most advanced area of the lesion, which requires one year of training

3. the ability to predict histopathology from the colposcopic pattern, a skill which may take many years.

False positive colposcopy occurs because of the failure to differentiate other non pathological causes of colposcopic changes to the cervical epithelium. These include 
physiological undifferentiated and differentiated metaplastic epithelium and sub clinical papilloma virus infection 39 . In addition, as already mentioned, the skill and experience of the colposcopist is central to accurate diagnosis.

In a series of 1410 patients reported by Stafl et al in 197341, the colposcopic prediction of histopathology obtained by punch biopsy was clinically accurate in $85 \%$ of cases. In only $3.3 \%$ of cases was the histopathology of the directed biopsy more advanced than predicted by colposcopy. However, in $11.7 \%$ of cases the histopathology was less advanced than expected colposcopically. This data correlates with a number of studies in which the high false positive rate of colposcopy is documented. 14,15

Critical to the interpretation of data about the accuracy of punch biopsy is knowledge of the degree of expertise of colposcopy. Our clinic is staffed by various levels of expertise and while junior staff are supervised, the practicalities of a very busy clinic may prevent all colposcopic examinations from being checked. This factor may account for the relatively high false positive rate of coloposcopy as indicated by the occurence of negative LLETZ in the look and LLETZ group. This factor was not specifically examined by this study.

This study was undertaken to evaluate the role of punch biopsy in reducing the negative LLETZ rate. Before considering the impact of punch biopsy on LLETZ histology an assessment of the reliability of punch biopsy is required. This study found that $25 \%$ of punch biopsies were falsely negative. This group of women required, on average, 3 clinic visits prior to definitive treatment, a considerable inconvenience to the patients. A negative punch biopsy provides false reassurance in this group of patients and further diagnostic procedures are essential to exclude CIN undetected by punch biopsy.

The sensitivity of punch biopsy was calculated at $86.36 \%$ indicating that the test is reasonably reliable in detecting the presence of CIN. The specificity is low at $61.81 \%$ confirming that the ability of punch biopsy to detect those without $\mathrm{CIN}$ is poor. 
The ability of punch biopsy to predict the presence of disease (positive predictive value) was high at $81.89 \%$. However the negative predictive value of punch biopsy was low at $68 \%$. While the calculation of the above data was based on the assumption that LLETZ histology is equivalent to two or more negative repeat smears, and therefore may be inaccurate, the results are consistent.

Accurate punch biopsy relies on:

1. Accurate localisation of the most abnormal area by prior colposcopy.

2. Correct usage of the punch biopsy forceps in order to obtain a representative sample and to avoid stripping of the dysplastic epithelium

3. Reliable interpretation of the findings by the histopathologist..

Studies that have compared colposcopically directed biopsy with a final diagnosis subsequently made on more extensive surgical procedures on the cervix including conisation of the cervix and LLETZ, have been contradictory.

In a study by Skehan et $\mathrm{al}^{22}$ in which histology of 118 cone biopsies was compared to previous colposcopically directed punch biopsies, the punch biopsy had a false negative rate of $54 \%$ and two cases of microinvasive carcinoma were not detected by punch biopsy.

In the study by Murdoch et al ${ }^{38} 27.4 \%$ of patients who had CIN 3 demonstrated on the final LLETZ histology and a further $9.5 \%$ who had $C I N 1$ or 2 , had colposcopically directed punch biopsies that were falsely negative.

In the study by Buxton et $\mathbf{a l}^{24}$, designed to determine the relationship between the histology of directed biopsy to the histology of the LLETZ specimen, in $54 \%(132 / 243)$ of cases, the results of the punch biopsy did not agree with those of the subsequent loop excision specimen. In $47 \%$ of the women a more severe lesion was found in the excised 
TZ which included three unsuspected adenocarcinomas in situ and one stage 1a(i) invasive squamous cancer. In addition, in 24 out of $50(48 \%)$ negative punch biopsies, CIN was diagnosed in the final LLETZ histology.

This contrasts with the study performed by Kirkup et al43 in which the colposcopically directed punch biopsy in 201 women was compared to histology obtained by more extensive operative procedures. In this series $98 \%$ of operative diagnoses were never more than one grade in advance of the diagnosis made by directed biopsy. In addition, in the seven patients with microinvasive or invasive carcinoma the diagnosis was suspected colposcopically and confirmed in the biopsy.

In a study by Veridiano et al44 in which the colposcopically directed punch biopsy was compared to subsequent surgical specimen in 643 patients, directed biopsy was accurate in $93 \%$ of cases. Only $0.3 \%$ of patients were significantly underdiagnosed, which included two cases of microinvasive carcinoma.

A falsely negative punch biopsy may be the result of either poor technique or a faulty instrument. Stripping of the abnormal epithelium occurs with ease making histological interpretation impossible. In addition the amount of tissue obtained may be too small to exclude microinvasion.

Histological interpretation is also influenced by observer variability, particularly in the grading of CIN.45,46 All specimens submitted by the colposcopy clinic at Groote Schuur Hospital are examined by the same two pathologists. It is doubtful therefore that variation in histological interpretation played a major role in introducing bias.

The next question to be addressed is the reliability of follow-up cytology in patients who were initially referred with cytology indicating $\mathrm{CIN}$. This study has identified a group of 31 patients who had negative punch biopsies and subsequently two negative smears at follow-up. This group of women were apparently saved an unnecessary surgical 
procedure, as all of them had CIN diagnosed cytologically and colposcopically initially.

The false negative rate of cytology is variously reported from $12-45 \%$ for CIN. 47.48 Errors may occur in sampling and in evaluation of the smears. Sampling errors are the most common cause for false negative cytology. All of our patients had repeat smears taken under ideal circumstances, that is in lithotomy position, with optimal exposure of the cervix and under colposcopic direction. In addition all samples were taken with both Aylesbury wooden spatula and the endocervical cytobrush. In all cases the smears contained endocervical and squamous cells and were judged satisfactory by the cytologist. It is therefore extremely unlikely that there was any sampling error in the follow-up cytology of these patients.

Furthermore a number of studies have documented a reduction in the false negative rate if smears are repeated 49,50 as they were in our study at three and six month intervals. While these patients require careful follow-up, the likelihood of missing a lesion is small.

When comparing the look and LLETZ group with those women who underwent LLETZ after a positive punch biopsy, the occurence of a negative LLETZ was statistically the same in each group, suggesting, that the intervention punch biopsy made no difference to the outcome. This data, however, must be interpreted with caution.

A number of factors could account for a negative LLETZ after a positive punch biopsy:

1. The histologist incorrectly interpreted the punch biopsy specimen and the result was falsely positive.

2. It is possible that the punch biopsy removed the entire focus of $\mathrm{CIN}$. In this study the size of the lesion was not routinely recorded and we have no way of knowing whether this could have occured. 
3. It has been suggested that performing a punch biopsy may alter the natural history of the dysplastic process in such a way that the lesion regresses spontaneously. 51 This is speculative and has not been addressed by this study. 


\section{CHAPTER 6}

\section{CONCLUSION}

This study has demonstrated that $18.01 \%$ of women had a negative LLETZ after a positive punch biopsy. This result is not statistically different from the $14.03 \%$ incidence of negative LLETZ in women treated with look and LLETZ.

However, $16.84 \%$ of women did not undergo LLETZ because of a negative punch biopsy, followed by persistently negative cytology. This group of women were 'saved' from a presumably unnecessary surgical procedure, LLETZ.

Punch biopsy does not reduce the occurence of negative LLETZ but it can be useful in the event of a negative punch biopsy with follow-up cytology. It must be emphasised that additional visits to the hospital for the results of the punch biopsy and repeat cytology, results in losing patients to follow-up. 


\section{REFERENCES}

1. Richart R.M., Barron B.A. A follow-up study of patients with cervical dysplasia. Am J Obstet Gynecol 1969;105:386

2. Galvin G.A., Jones H.A., Te Linde R. W. The significance of basal cell hyperactivity in cervical biopsies. Am J Obstet Gynecol 1955;70:808

3. McKay D.G., Terjanian B., Poschyachinda D. et al. Clinical and Pathologic Significance of Anaplasia (Atypical Hyperplasia) of the Cervix Uteri. Obstet Gynecol 1959;13:2-21

4. Fox C.H. Biologic behaviour of dysplasia and carcinoma in situ. Am J Obstet Gynecol 1967;99:960

5. Boyes D.A., Morrison B., Knox E.G. A cohort study of cervical cancer screening in British Columbia. Clin Invest Med 1982;5:1-29

6. Johannesson G., Geirsson G., Day N. The effect of mass screening in Iceland 1965-74 on the incidence and mortality of cervical carcinoma. Int $\mathrm{J}$ Cancer $1978 ; 21: 418-425$

7. Jones H.W., Buller R.E. The treatment of cervical intraepithelial neoplasia by cone biopsy. Am J Obstet Gynecol 1980;137:882

8. Jones J.M., Sweetnam P., Hibbard B.M. The outcome of pregnancy after cone biopsy of the cervix. A case control study. Br J Obstet Gynaecol 1979;86:913

9. Nebel W., Shingelton H.M., Swanton M.C. Cold knife conisation of the cervix uteri. Surg Gynaecol Obstet 1967;125:780.

10. McCann S.W., Mickal A., Crapanzano J.T. Sharp Conisation of the Cervix. Observations of 501 consecutive patients. Obstet Gynecol 1969;33:470-475

11. Krumholz B.A., Knapp R.C. Colposcopic Selection of Biopsy Sites. Obstet Gynecol 1972;39:22-26

12. Anderson S.G., Linton E.B. The diagnostic accuracy of cervical biopsy and cervical conisation. Am J Obstet Gynecol 1967;99:113 
13. Silbar E.L., Woodruff J.D. Evaluation of biopsy, cone and hysterectomy sequence in intraepithelial carcinoma of the cervix. Obstet Gynecol 1966;27:89

14. Limburg $\mathrm{H}$. Comparison between cytology and colposcopy in the diagnosis of early cervical carcinoma. Am J Obstet Gynecol 1958;75:1295

15. Navratil E., Burghardt E., Bajardi F., Nash W. Simultaneous colposcopy and cytology used in screening for carcinoma of the cervix. Am J Obstet Gynecol $1958 ; 75: 1292$

16. Crapanzano J.T. Office diagnosis in patients with abnormal cervicovaginal cytosmears: Correlation of colposcopic biopsy with cytologic findings. Am J Obstet Gynecol 1972;113:967

17. Jordan J.A. The treatment of cervical intraepithelial neoplasia by laser vaporisation. $\mathrm{Br} \mathrm{J}$ of Obstet Gynaecol 1985;92:394-398

18. Goldberg G.L., Bloch B., Edwards J.T. et al. Carbon dioxide laser surgery for cervical intraepithelial neoplasia. SAMJ 1985;68:758

19. Anderson M.C. Treatment of Cervical Intraepithelial Neoplasia with the Carbon Dioxide Laser: Report of 543 Patients. Obstet Gynecol 1982;59:720

20. Ali S.W., Evans A.S., Monaghan J.M. Results of $\mathrm{CO}_{2}$ laser cylinder vaporisation of cervical intraepithelial disease in 1234. An analysis of failures. $\mathrm{Br} J$ Obstet Gynaecol 1986;93:75-78

21. Carter P.G., Harris V.G., Wilson P.O.G. The management of the abnormal cervical smear: a comparative study between loop diathermy alone and laser ablation preceded by colposcopically directed punch biopsy. J Obstet Gynaecol 1993:13:59-63

22. Skehan M., Soutter W.P., Lim K. et al. Reliability of colposcopy and directed punch biopsy. Br J Obstet Gynaecol 1990;97:811-816

23. Howe D.T., Vincenti A.C. Is Large Loop Excision of the Transformation Zone (LLETZ) more accurate than colposcopically directed punch biopsy in the diagnosis of cervical intraepithelial neoplasia? Br J Obstet Gynaecol 1991;98:588-591 
24. Buxton E.J., Luesley D.M., Shafi M.I., Rollason M. Colposcopically directed punch biopsy: a potentially misleading investigation. Br J Obstet Gynaecol 1991;98:1273 $-1276$

25. Anderson M.C. Invasive carcinoma following local treatment for $\mathrm{CIN}$. Br J Obstet Gynaecol 1993;100:657-663

26. Anderson M.C., Hartley R.B. Cervical crypt involvement by intraepithelial neoplasia. Obstet Gynecol 1980;55:546

27. Abdul-Karim F.W., Nunez C. Cervical intraepithelial neoplasia after conisation: a study of 522 consecutive cervical cones. Obstet Gynecol 1985;65:77

28. Coppleson M., Atkinson K.H., Dalrymple J.C. Cervical squamous and glandular intraepithelial neoplasia: clinical features and review of management. In Gynecologic Oncology. Coppleson M. et al (eds). "Churchill Livingstone. Edinburgh. Second Edition Volume 1 pg 1992;593-597

29. Choo Y.C., Chan O.L.Y., Hsu C., Ma H.K. Colposcoy in micro-invasive carcinoma of cervix - an enigma of diagnosis. Br J Obstet Gynaecol 1984;91:1156-1160

30. McIndoe G.A.J., Robson M.S., Tidy J.A., Mason W.P., Anderson M.C. Laser Excision Rather Than Vaporisation: The Treatment of Choice for Cervical Intraepithelial Neoplasia. Obstet Gynecol 1989;74:165-168

31. Cartier R. 1981 Use of the diathermy loop in the diagnosis and treatment of lesions of the uterine cervix. Colposcopy of the connective tissue. Fourth World Congress, International Federation for Cervical Pathology and Colposcopy. London

32. Prendiville W., Davies R., Berry P.J. A low voltage diathermy loop for taking cervical biopsies: a qualitatitve comparison with punch biopsy forceps. $\mathrm{Br} \mathrm{J}$ Obstet Gynecol 1986;93:773-776.

33. Prendiville W., Cullimore J., Norman S. Large Loop Excision of the Transformation Zone (LLETZ). A new method of management for women with cervical intraepithelial neoplasia. Br J Obstet Gynaecol 1989;96:1054-1060. 
34. Luesley D.M., Cullimore J., Redman C.W.E. et al. Loop diathermy excision of the cervical transformation zone in patients with abnormal cervical smears. $\mathrm{Br}$ Med $\mathrm{J}$ 1990;300:1690

35. Oyesanay O.A., Amerasinghe C.N., Manning E.A.D. Outpatient excisional management of cervical intraepithelial neoplasia. Am J Obstet Gynecol 1993; $168: 485$

36. Bigrigg M.A., Codling B.W., Pearson P. et al. Colposcopic diagnosis and treatment of cervical dyspasia at a single clinic visit. Experience of low-voltage diathermy loop in 1000 patients. Lancet 1990;336:229-31

37. Keijser G.G., Kenemans P., H.Th.H van der Zanden P. Diathermy loop excision in the management of cervical intraepithelial neoplasia: Diagnosis and treatment in one procedure. Am J Obstet Gynecol 1992;166:1281

38. Murdoch J.B., Grimshaw R.N., Monaghan J.M. Loop diathermy excision of the abnormal cervical transformation zone. Int J Gynaecol Cancer 1991;1:105 - 111

39. Coppleson M., Pixley E.C. 1992 Colposcopy of the Cervix. In Gynecologic Oncology. Coppleson M. et al (eds). Churchill Livingstone. Edinburgh. Second Edition Vol 1 pg 318

40. Stafl A. 1976b Colposcopy. Cancer (suppl 1) 38:432

41. Stafl A., Mattingly R.F. Colposcopic Diagnosis of Cervical Neoplasia. Obstet Gynecol 1973;41:168

42. Kennedy S., Robinson J., Hallam N. LLETZ and Infertility. Br J Obstet Gynaecol 1993;100:965

43. Kirkup W., Hill A.S. The Accuracy of Colposcopically Directed Biopsy in Patients with Suspected Intraepithelial Neoplasia of the Cervix. $\mathrm{Br} J$ Obstet Gynaecol $1980 ; 87: 1-4$

44. Veridiano N.P., Delke I. Tancer M.L. Accuracy of colposcopically directed biopsy in patients with cervical neoplasia. Obstet Gynecol 1981;58:185

45. Robertson A.J., Anderson J.M., Swanson Beck J. et al. Observer variablility in histopathological reporting of cervical biopsy specimens. J Clin Pathol $1989 ; 42 ; 231-238$ 
46. Fox H., Buckley C.H. Current problems in the pathology of intraepithelial lesions of the uterine cervix. Histopathology 1990;17:1-6

47. Richart R.M. 1964 Evaluation of the true false negative rate in cytology. Am J Obstet Gynecol July 15.

48. Koss L. The Papanicolaou Test for Cervical Cancer Detection. A Triumph and a Tragedy. JAMA 1989;261:737

49. Davis J.R., Hindman W.M., Paplanus M.D. Value of Duplicate smears in Cervical Cytology. Acta Cytol 1981;25: 533

50. Luthy D.A., Briggs R.M., Buyco A. et al. Cervical Cytology; Increased sensitivity with a second cervical smear. Obstets Gynecol 1978;51:713-717

51. Richart R.M. Natural history of cervical intraepithelial neoplasia. Clin Obstet Gynecol 1973;10:748 